\title{
Correlation between Cytoplamic Oocyte Maturation and Chromo- somal Aneuploidies - Impact on fertilization, embryo quality and pregnancy
}

\author{
Cristian Alvarez Sedó ${ }^{1}$, Mariana Miguens ${ }^{1}$, Sara Andreucci ${ }^{1}$, Noelia Ortiz ${ }^{1}$, Daniela Lorenzi ${ }^{1}$, Sergio Papier ${ }^{1}$, \\ Florencia Nodar ${ }^{1}$ \\ ${ }^{1}$ Centro de Estudios en Genética y Reproducción (CEGYR) - Buenos Aires, Argentina
}

\begin{abstract}
Objective: To establish the relationship between oocyte cytoplasmic maturation and its chromosomal status and determine the effect of this feature over the reproductive outcome in patients with sub-optimal fertilization in ART.

Methods: Fifty couples who underwent ART were selected. From nineteen patients, 22 metaphase II-MII and 18 failed-fertilized oocytes after ICSI were studied. The first polar body was collected for chromosomal analysis by aCGH. Oocytes were processed by immunocytochemistry (ICC) to determine oocyte maturation: assessment of inactive MPF status and the conformation-alignment of the metaphase plate.

Other 31 couples presented sub-optimal fertilization $(<50 \%)$ after ICSI, and failed-fertilized oocytes were studied by ICC. Two groups were conformed according to the main feature observed: A) cytoplasmic immaturity and sperm premature chromosome condensation and B) sperm nuclear decondensation failure with mature cytoplasm.

Results: Regarding MII mature oocytes, $87 \%$ had a normal metaphase plate and $84 \%$ were chromosomally normal. Contrary, immature oocytes presented abnormal metaphase plate $(86 \%)$ and just $33 \%$ were euploid. In failed-fertilized oocytes: $100 \%$ of mature oocytes had a normal metaphase plate and $71 \%$ were euploid. When oocytes were cytoplasmic immature, $37 \%$ of them were normal (metaphase plate) and 50\% were chromosomally normal.

The global rate of aneuploidies and metaphase plate disarrangements in immature oocytes (MII+failed-fertilized) were significantly higher than mature oocytes $(P<0.05)$.

In patients with sub-optimal fertilization, the percentage of top quality embryos and pregnancy rate was significantly higher in group $\mathrm{B}(P<0.05)$.

Conclusion: Oocyte cytoplasmic immaturity is related to metaphase plate anomalies and aneuploidies. Fertilized oocytes, from a cohort with sub optimal fertilization with cytoplasmic immaturity, had poorer reproductive outcomes.
\end{abstract}

Keywords: Cytoplasmic maturation, MPF, metaphase plate, aneuploidies.

\section{INTRODUCTION}

Nuclear and cytoplasmic oocyte maturation occurs in vivo during follicular growth and ovulation. Both processes are induced by changes in plasma levels of gonadotropins. The relationship between the oocyte and surrounding follicular cells is modified during maturation period as a result of changes in the oocyte membrane and in the intracellular signaling system (Moor et al., 1990; Thibault, 1977).

The extra-nuclear maturation involves changes in cytoplasmic organelles organization, in the cell membrane and in the vitelline matrix (Downs 1990; Downs 1993). Nuclear maturation is characterized by alternating states of development and arrest of meiosis. The first interruption during meiosis is at the germinal vesicle stage (GV). The GV helps in a practical way for identifying immature oocytes. During their growth, oocytes acquire the ability to restart meiotic maturation in response to gonadotropins stimulation, mainly LH increased. The nuclear envelope breaks down, the first polar body is released and meiosis progress until metaphase II (MII). The meiosis stops in MII and ovulation occurs. The MII stage is maintained until fertilization or parthenogenetic activation of the oocyte, when the second polar body is released.

In mammals, maturation promoting factor (MPF) and mitogen activated protein kinases (MAPK) play an important role in oocyte maturation. Regarding MAPK, its activity allows the adequate meiosis progression and MII arrest after extrusion of the first polar body (Combelles et al., 2005). MPF, as described in the early 70s, is responsible for meiosis resumption of oocytes arrested at prophase stage (Masui \& Markert, 1971). MPF is constituted by a catalytic subunit, cyclin dependent kinase (cdc2) and a regulatory subunit, Cyclin B. Cyclin B concentration varies during cellular cycle. The amount of MPF molecules depends on the Cyclin B availability in the cytoplasm able to conjugate with cdc2, which its concentration maintained during cellular cycle in the oocyte. MPF concentration increased during oocyte maturation, reaching maximum peak during MII stage. The complex suffers a series of phosphorylation and dephosphorylation. Thus, when cdc2 is phosphorylated in threonine 161 residue (T161) the complex is activated. This occurs from Anaphase I - Telophase I (AI-TI) to MII stage. On the other hand, if the cdc2 is phosphorylated in tyrosine 15 residue (Y15), the system is inactive. MPF activation is self-regulated by a positive feed-back from MPF activated molecules that increased in the oocyte (Ferrell, 1999). Furthermore, MPF activates associated microtubules proteins such as MAPK (Whitaker, 1996).

After maturation, MPF reaches the highest concentration and the mature oocyte is arrested at MII. This arrest stage is exclusive for oocytes and is regulated by the cytostatic factor (CSF). This complex is in charge of stabilizing the MPF, maintaining chromosomes condensed and avoiding a second round of DNA replication during transition from metaphase I (MI) to MII (Verlhac et al., 1994; Sagata, 1996).

In humans, the possibility of obtaining a higher number of MII oocytes through hormonal controlled ovarian stimulations has allowed the increase in the number of embryos obtained through in vitro fertilization (IVF) at assisted reproductive treatments (ART), increasing the chances of reproductive success. The implementation of ICSI has also helped ART in cases of severe male factor.

When in vivo or in vitro fertilization occurs, it is assumed that both gametes have the essential elements for an adequate fertilization. The sperm requires some important proteins, such as phospholipase $\mathrm{C}$ zeta, for the reactivation of the oocyte arrested at MII stage through the induction of intracellular calcium pulses. The correct activation of the oocyte allows the restart of meiosis II, the release of the second polar body and the pronuclear formation. However, 
Figure 1. Flux diagram for the assessment of metaphase II and failed-fertilized oocytes.

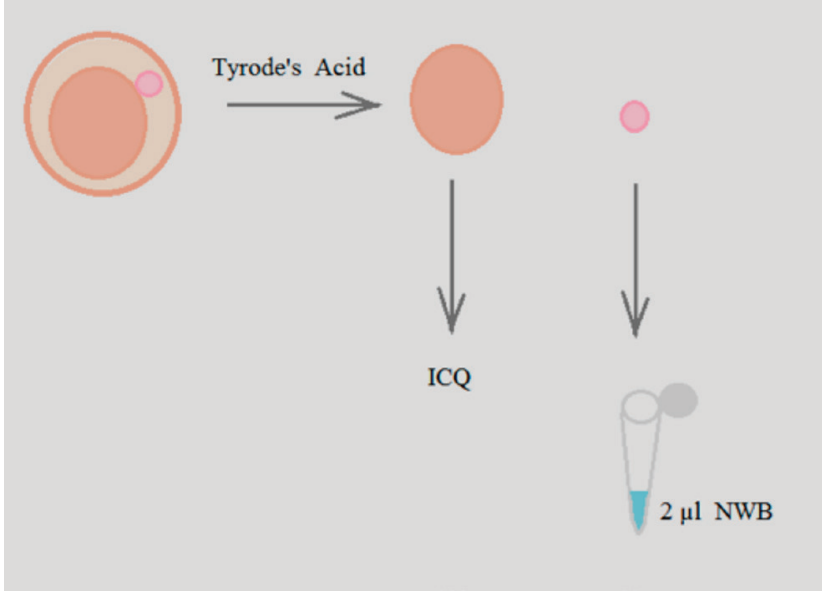

Figure 2. Agarose electrophoretic gel (2\%) for polar body DNA amplification detection. Positive control and polar bodies (orange), negative control (color less).

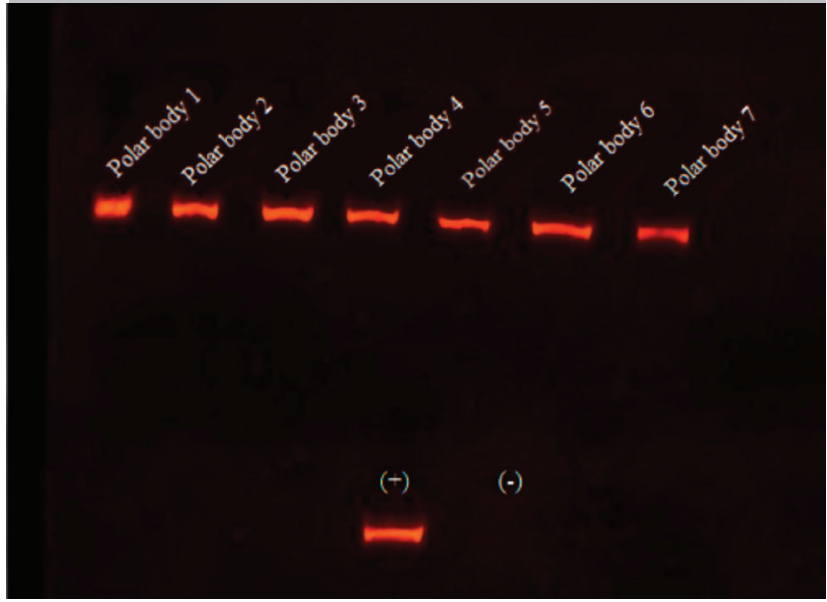

Figure 3. aCGH profiles. Normal or euploid oocyte $(A)$, abnormal or aneuploid oocyte (B) (trisomy = blue, monosomy $=$ red).

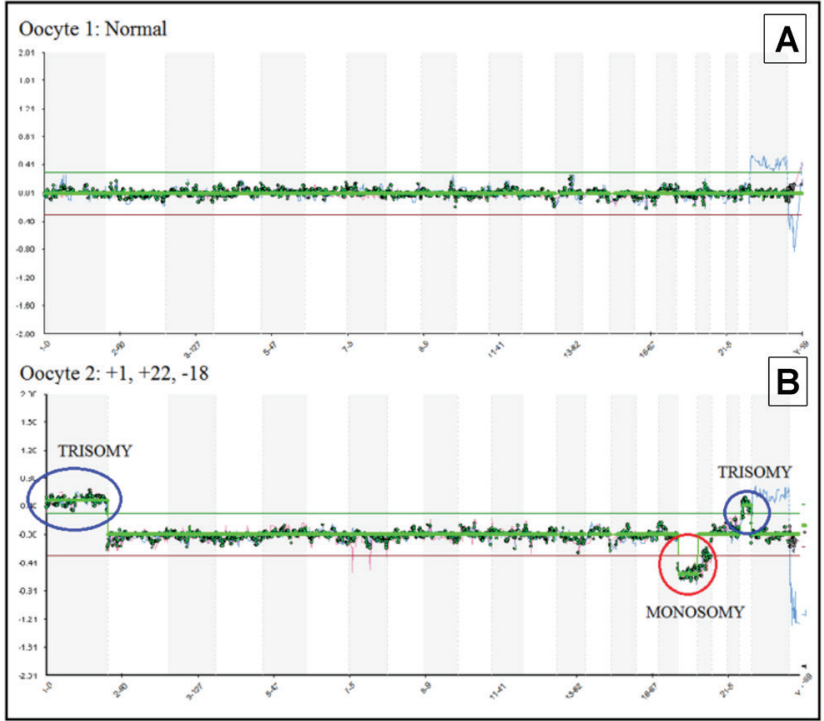

Figure 4. $\mathrm{pCdc} 2(\mathrm{Y} 15)$ expression during oocyte maturation: GV stage (A, E and F), MI-TI ( $B, E$, and F), MII ( $C$, $E$, and $F$ ), and failed-fertilized oocyte depicting premature chromosome condensation (PCC) with high levels of pCdc2 (Y15) (immature).

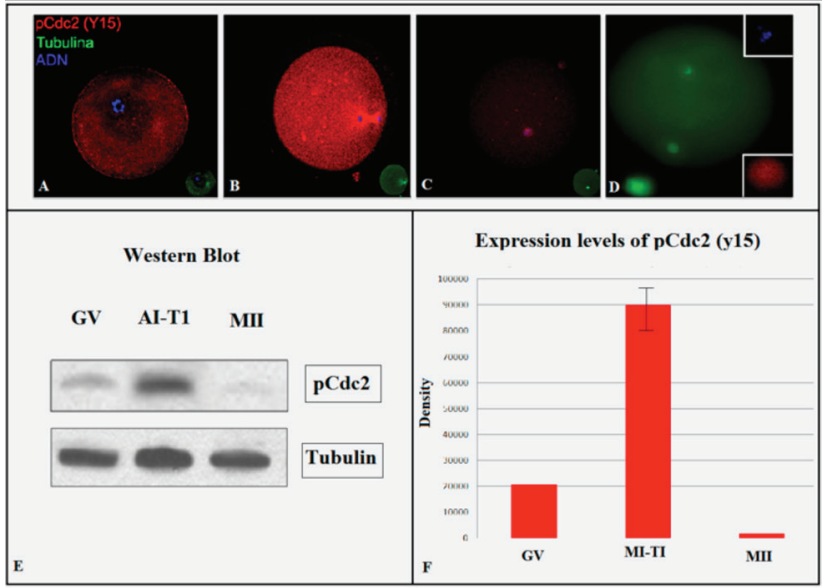

Figure 5. Left: normal metaphase plate conformation and correct chromosomal alignment. Right: abnormal metaphase plate conformation with chromosomal disarrangements.
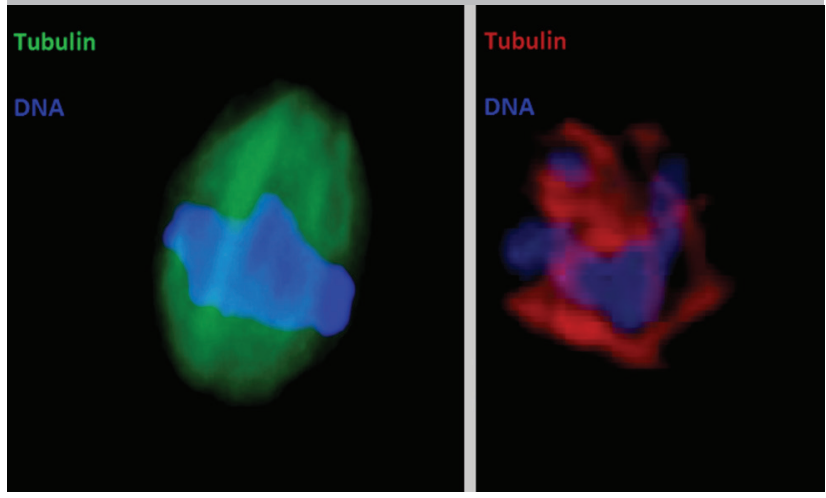

fertilization failure in ART is not an isolated event, principally as a result of oocyte activation failure. This result can be associated with two different events: sperm nuclear descondensation failure and premature chromosome condensation (PCC). Both have been described as the principal induction events in fertilization failure studies.

It is important to determine the importance of adequate nuclear and cytoplasmic oocyte maturation and it responsibility on fertilization. The aim of this study was to establish the relationship between oocyte cytoplasmic maturation and its chromosomal status by the assessment of the first polar body. Also, it was important to determine the effect of the oocyte cytoplasm maturation state over the reproductive outcome in ART patients with suboptimal fertilization rate.

\section{MATERIAL AND METHODS \\ Population}

Fifty couples who underwent to ART procedures were selected for this study (2013-2014). Thirty-one of them presented sub-optimal fertilization $(<50 \%)$ after ICSI. All women were considered as normal responders $(<37$ years old, antral follicle count $>10, \mathrm{FSH}<10 \mathrm{IU} / \mathrm{I}$ ). Patients with severe male factor were excluded. There is no conflict of interest at the present study. 
Figure 6. aCGH profiles. Top panel: Chromosomally normal oocyte and polar body. Bottom panel: Aneuploid oocyte and polar body (chromosome 16, 22).
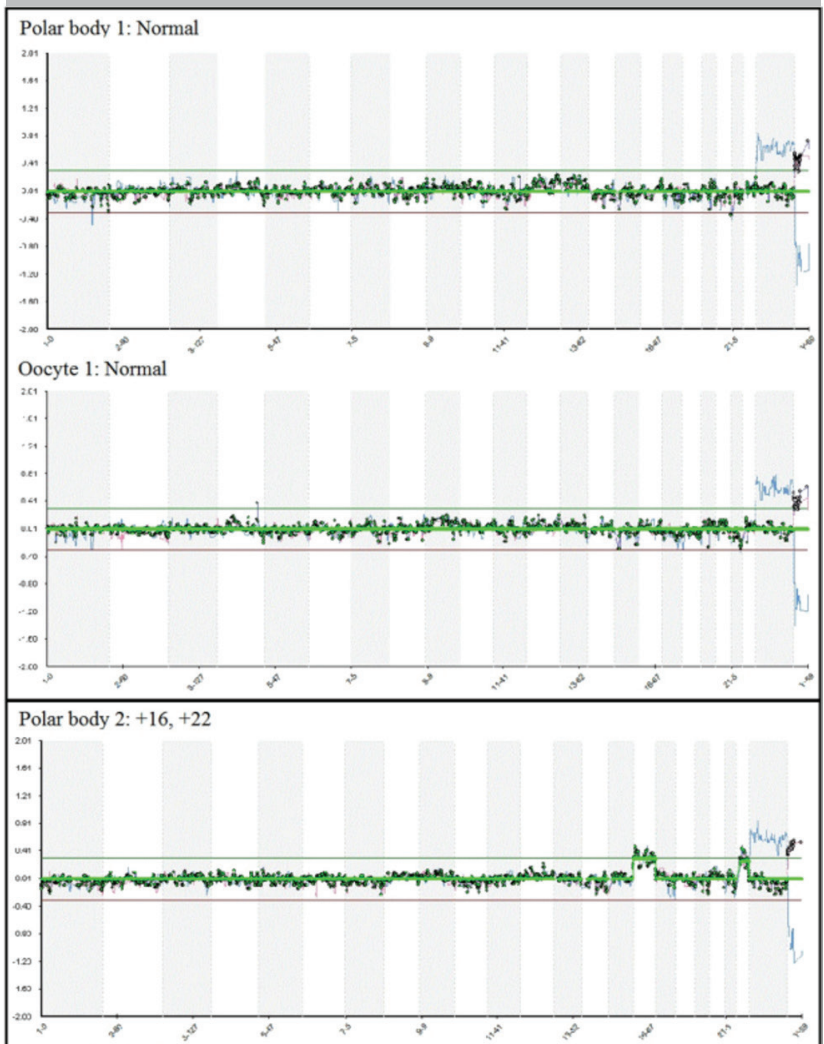

Oocyte 2: $-16,-22$

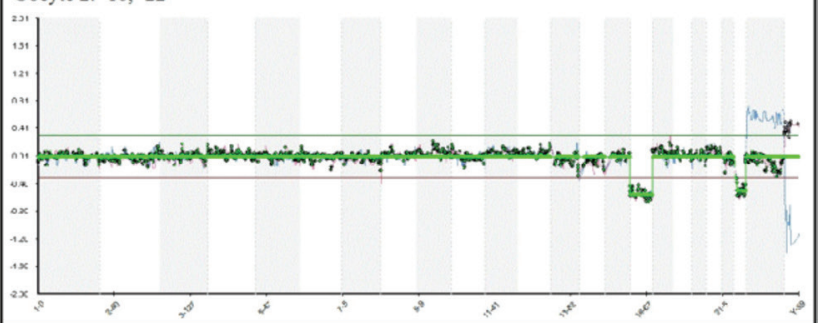

\section{Controlled ovarian stimulation}

All patients received flexible GnRH antagonist protocol for ovarian stimulation. Doses of gonadotrophin were started on day 3 in which the patient received $300 \mathrm{IU}$ ( $\mathrm{rFSH}$ ) (Gonal, Merck Serono, Italy) daily i.m. until the day of the HCG injection. When the leading follicle reached a diameter of $14 \mathrm{~mm}, \mathrm{GnRH}$ antagonist, cetrorelix (Cetrotide, Merck Serono, Germany) 0.25 mg daily was administered s.c. into the lower abdominal wall until the day of hCG injection. Once the leading follicle reached $17-18 \mathrm{~mm}$ in diameter with estradiol concentration $>+500 \mathrm{pg} / \mathrm{ml}$, hCG 10000 IU (Gonacor; Ferring, Argentina) was administered i.m 36 hours prior to oocyte retrieval. After that, an IVF procedure (ICSI) was performed as usually.

\section{Source of oocytes}

All human oocytes were donated for research with informed written consent by couples undergoing assisted reproduction procedures at CEGyR (Buenos Aires, Argentina). All human procedures were approved by the Internal Review Board and Ethics Committee of CEGyR. None of the presumed normal ova donated for this project were allowed to cleave in vitro or transferred to recipients after ICSI. These ova were going to be discarded if not donated for research.

All oocytes were used immediately upon oocyte retrieval by ultrasound guided transvaginal aspiration $36 \mathrm{~h}$ post-hCG administration. Failed-to-fertilize oocytes (no pronuclei visualized $18-20 \mathrm{~h}$ after sperm injection) were obtained $30-35 \mathrm{~h}$ post oocyte retrieval following ICSI. Oocytes recovered with inferior morphology were excluded from the study.

\section{Chemicals and antibodies}

All chemicals were obtained from Sigma Chemical Co. (St. Louis, MO, USA), unless otherwise stated. Inactive MPF (pCdc2-Y15), acetylated tubulin and tubulin were recognized using anti-full-length human pCdc2-Y15 (rabbit monoclonal, dil: 1:100, Cell Signaling, USA), acetylated tubulin (mouse monoclonal, dil: 1:100, Sigma Aldrich, USA), anti-tubulin antibody (dil: 1:200, Cytoskeleton, US), respectively. Secondary Alexa Fluor antibodies were obtained Molecular Probes (Invitrogen, US).

Table 1. Results of maturation state, metaphase plate conformation and euploidy assessment at MII and failed-fertilized

\begin{tabular}{|c|c|c|c|c|}
\hline & $\begin{array}{c}\text { Cytoplasmic maturation } \\
\text { state (MPF) }\end{array}$ & $\begin{array}{l}\text { MII structural } \\
\text { conformation }\end{array}$ & \multicolumn{2}{|c|}{$\begin{array}{c}\text { PB normal } \\
\text { ploidy (aCGH) }\end{array}$} \\
\hline \multirow{4}{*}{22 MII oocytes } & \multirow[b]{2}{*}{$\begin{array}{l}\text { Mature } \\
\quad 15\end{array}$} & $\begin{array}{c}\text { Normal } \\
13(87 \%) *, * *\end{array}$ & $\begin{array}{l}11 / 13 \\
(84 \%)\end{array}$ & \multirow[b]{2}{*}{$\begin{array}{l}12 / 15 \\
(80 \%)\end{array}$} \\
\hline & & $\begin{array}{c}\text { Abnormal } \\
2(13 \%)\end{array}$ & $\begin{array}{c}1 / 2 \\
(50 \%)\end{array}$ & \\
\hline & \multirow[b]{2}{*}{$\begin{array}{c}\text { Immature } \\
7\end{array}$} & $\begin{array}{c}\text { Normal } \\
1(14 \%)^{*}\end{array}$ & $\begin{array}{c}0 / 1 \\
(0 \%)\end{array}$ & \multirow[b]{2}{*}{$\begin{array}{c}2 / 7 \\
(29 \%)\end{array}$} \\
\hline & & $\begin{array}{l}\text { Abnormal } \\
6(86 \%)\end{array}$ & $\begin{array}{c}2 / 6 \\
(33 \%)\end{array}$ & \\
\hline \multirow{4}{*}{$\begin{array}{l}18 \text { failed-fertilized } \\
\text { oocytes (OPN) }\end{array}$} & \multirow[b]{2}{*}{$\begin{array}{c}\text { Mature } \\
7\end{array}$} & $\begin{array}{c}\text { Normal } \\
7(100 \%) *, * *\end{array}$ & $\begin{array}{c}5 / 7 \\
(71 \%)\end{array}$ & \multirow[b]{2}{*}{$\begin{array}{c}5 / 7 \\
(71 \%)\end{array}$} \\
\hline & & $\begin{array}{l}\text { Abnormal } \\
0(0 \%)\end{array}$ & $\begin{array}{l}0 / 0 \\
(0 \%)\end{array}$ & \\
\hline & \multirow[b]{2}{*}{$\begin{array}{c}\text { Immature } \\
11\end{array}$} & $\begin{array}{l}\text { Normal } \\
4(37 \%)\end{array}$ & $\begin{array}{c}2 / 4 \\
(50 \%)\end{array}$ & \multirow[b]{2}{*}{$\begin{array}{c}4 / 11 \\
(36 \%)\end{array}$} \\
\hline & & $\begin{array}{l}\text { Abnormal } \\
7(63 \%)\end{array}$ & $\begin{array}{c}2 / 7 \\
(29 \%)\end{array}$ & \\
\hline
\end{tabular}


Table 2. Global results of maturation state, metaphase plate conformation and euploidy assessment at MII and failed-fertilized oocytes.

\begin{tabular}{|c|c|c|}
\hline $\begin{array}{c}\text { Cytoplasmic } \\
\text { maturation } \\
\text { state (MPF) }\end{array}$ & $\begin{array}{c}\text { Normal MII } \\
\text { structural } \\
\text { conformation }\end{array}$ & $\begin{array}{c}\text { PB normal } \\
\text { ploidy } \\
\text { (aCGH) }\end{array}$ \\
\hline Mature & $20 / 22$ & $17 / 22$ \\
22 & $(91 \%)^{*}$ & $(77 \%)^{*}$ \\
\hline $\begin{array}{c}\text { Immature } \\
18\end{array}$ & $5 / 18$ & $6 / 19$ \\
$(27.8 \%)$ & $(32 \%)$ \\
\hline
\end{tabular}

\section{Oocytes processing}

From nineteen patients, 22 metaphase II and 18 failed-fertilized oocytes after ICSI were collected and studied. All oocytes were individually processed. Zona pellucida was dissolved by incubation in Tyrode's acid (Irvine Scientific, US) and the first polar body was collected washed in Non-sticking Washing Buffer (NWB) and then introduced in a $0.2 \mathrm{~mL}$ PCR tube containing $2 \mathrm{uL}$ of NWB for aCGH (comprehensive genome hybridization - array) procedure (Figure 1). Then the oocytes were fixed and processed by immunocytochemistry (ICC).

The other 31 couples, presented sub-optimal fertilization after ICSI, and the failed-fertilized oocytes were separated and studied for ICC.

\section{Polar body chromosomal analysis by aCGH}

Negative controls, consisting of $2 \mathrm{uL}$ of Non-sticking Washing Buffer (NWB) taken from drops used for washing the biopsied cells, were placed in separate tubes. The samples were lysed, fragmented, and amplified using the SurePlex kit, according to the manufacturer's instructions (BlueGnome, UK). One nanogram of genomic DNA and one reagent negative control (amplification mixture only) were also subjected to whole genome amplification (Figure 2).

The samples and reference male DNAs were lysed fragmented and amplified using the SurePlex whole genome amplification kit (BlueGnome, Cambridge, UK) according to the manufacturer's instructions. Amplified DNA samples and reference male DNA were labelled with Cy3 and Cy5, respectively, using the BlueGnome fluorescent labelling system, according to the manufacturer's instructions. Amplification and labelling protocols used in this study are available at www.cytochip.com. The platform used in this study was the 24Sure Cytochip (BlueGnome), which screens all 24 chromosomes for both gain and loss with a BAC pooling strategy together with software smoothing which en- ables robust results to be reported on the ploidy of the single cell on the basis of a single hybridization. Labelled samples and reference male DNAs were mixed and applied to a microarray and co-hybridized for a minimum of $3 \mathrm{~h}$, after which they were washed in $\cdot 2$ saline sodium citrate (SSC)/0.05\% Tween 20 at room temperature for $10 \mathrm{~min}$, followed by a wash in $1 \mathrm{SSC}$ at room temperature for $10 \mathrm{~min}$ and with $0.1 \mathrm{SSC}$ at $59 \mathrm{C}$ for $5 \mathrm{~min}$ and finally washed for $1 \mathrm{~min}$ at room temperature in the same solution. Microarray slides were dried in a centrifuge for 3 min and scanned with a laser scanner (InnoScan 710AL; Innopsys, Carbonne, France). Scanned images were analysed using BlueFuse Multi software (BlueGnome). Once a specific amplification was observed, autosomal profiles were analysed for gain or loss of whole or partial chromosomal ratios using a 3SD assessment, $0.3 \log 2$ ratio call or both. To pass hybridization quality controls, sex mismatched female samples had to show a consistent gain on chromosome $X$ and a consistent loss of chromosome $Y$. Sex-matched male samples had to consistently show no change on either chromosome $\mathrm{X}$ or $\mathrm{Y}$ (Gutiérrez-Mateo et al., 2011). Oocytes were classified as 'normal' if the generated array CGH plot showed no gains or losses in any of the autosomes, 'aneuploid' if gains or losses were observed for any chromosome (Figure 3 ).

\section{Immunocytochemistry for oocyte maturation as-} sessment

Zona-free human oocytes were then fixed for $40 \mathrm{~min}$ in $2 \%$ formaldehyde and permeabilized in PBS $+0.1 \%$ Triton $\mathrm{X}-100$ for an additional 40 min (method modified and based on Messinger and Albertini, 1991). After fixation and permeabilization, samples were blocked for $1 \mathrm{~h}$ in PBS + $0.3 \%$ bovine serum albumin (BSA) $+1 \%$ fetal calf serum prior to incubation in humidified chambers with primary and secondary antibodies, overnight at $4^{\circ} \mathrm{C}$ and for $1 \mathrm{~h}$ at room temperature, respectively.

Samples were counterstained for DNA using Hoechst 33258. Images were obtained using an Olympus spectral confocal microscope, with laser lines at 488-, 568- and 633 -nm wavelengths and then processed using Adobe Photoshop C5. Negative controls were run in the absence of primary antibodies. This assay allows to us to determine the cytoplasmic maturation state of the oocyte by the assessment of the inactive form of the MPF complex and also the conformation and alignment of the metaphase plate (Figure 4 and 5).

\section{Statistics}

The Student's t-test was used for between-group comparisons, and the Mann-Whitney U-test was used to as-

Table 3. Comparison between groups A and B regarding male and female reproductive characteristics - Population of ART patients with suboptimal fertilization.

\begin{tabular}{|c|c|c|c|}
\hline & Group A & Group B & $\boldsymbol{P}$ \\
\hline $\mathrm{N}$ & 13 & 18 & \\
\hline Male age & $37.1 \pm 3.3$ & $36.6 \pm 3.5$ & NS \\
\hline Sperm morphology & $7.2 \pm 3.9$ & $8.5 \pm 3.1$ & NS \\
\hline Female age & $34.5 \pm 2.6$ & $35.6 \pm 2.1$ & NS \\
\hline Duration of infertility (years) & $4.1 \pm 2.7$ & $3.9 \pm 2.5$ & NS \\
\hline AFC & $17.1 \pm 4.1$ & $16.2 \pm 5.4$ & NS \\
\hline $\mathrm{FSH}(\mathrm{mIU}(\mathrm{mL})$ & $6.6 \pm 1.3$ & $6.6 \pm 1.7$ & NS \\
\hline $\mathrm{LH}(\mathrm{mIU} / \mathrm{mL})$ & $4.5 \pm 2.4$ & $4.3 \pm 1.2$ & NS \\
\hline Total rFSH dose & $2400 \pm 420$ & $2500 \pm 410$ & NS \\
\hline $\mathrm{N}^{\circ}$ days of ovarian stimulation & $8.9 \pm 1.3$ & $8.5 \pm 0.9$ & NS \\
\hline
\end{tabular}


Table 4. Comparison between groups $A$ and $B$ regarding MII oocytes retrieved and fertilization rate.

\begin{tabular}{|c|c|c|c|}
\hline & Group A & Group B & $\boldsymbol{P}$ \\
\hline $\begin{array}{c}\text { No of oocytes } \\
\text { retrieved }\end{array}$ & $13.8 \pm 4.3$ & $12.9 \pm 3.5$ & NS \\
\hline $\begin{array}{c}\text { No of MII } \\
\text { oocytes }\end{array}$ & $10.5 \pm 3.5$ & $9.9 \pm 3.1$ & NS \\
\hline$\%$ MII retrieval & $77.3 \pm 12.9$ & $77.3 \pm 12.9$ & NS \\
\hline$\%$ 2PN & $30.0 \pm 13.5$ & $31.5 \pm 10.5$ & NS \\
\hline \% NoPN & $62.1 \pm 21.6$ & $62.5 \pm 7.5$ & NS \\
\hline Others & $7.9 \pm 6.9$ & $6.0 \pm 6.2$ & NS \\
\hline
\end{tabular}

Table 5. Reproductive outcomes between groups A and B.

\begin{tabular}{|c|c|c|c|}
\hline & Group A & Group B & $\boldsymbol{P}$ \\
\hline $\begin{array}{c}\text { \% Top quality } \\
\text { embryos (48 hs) }\end{array}$ & 22.2 & 37.3 & 0.03 \\
\hline $\begin{array}{c}\mathrm{N}^{\circ} \text { of embryo } \\
\text { transferred/cycle }\end{array}$ & 2.0 & 1.9 & $\mathrm{NS}$ \\
\hline $\begin{array}{c}\text { Clinical pregnan- } \\
\text { cy (\%) }\end{array}$ & 12.1 & 34.4 & 0.001 \\
\hline
\end{tabular}

sess homogeneity. Differences were considered significant when $P<0.05$. MedCalc 12.4 software (Belgium) was used for the statistical analysis.

\section{RESULTS}

The results of the cytoplasmic oocyte maturation assessment, the metaphase plate conformation and the chromosomal status of the polar body ( 22 metaphase II and 18 failed-fertilized oocytes) is shown in table 1 and 2. As verification that the euploidy-aneuploidy observed at polar body was a representative of the euploidy status, aCGH procedure was conducted in some oocytes and their corresponding 1st polar body. Figure 6 depicts the complementary status of the chromosomal number.

The immaturity of the oocyte cytoplasm is related to abnormalities at the metaphase plate conformation and chromosome number (increased aneuploidy index). Considering the metaphase II oocytes, when nuclear and cytoplasmic maturation occurred, $87 \%$ of them had a normal metaphase plate conformation and $84 \%$ were chromosomally normal. Contrary, when the oocyte depicted immaturity signals, $86 \%$ had an abnormal metaphase plate conformation and just 33\% were euploid. Regarding to the failed-fertilized oocytes, the observed pattern was similar, $100 \%$ of the mature oocytes had a normal metaphase plate and $71 \%$ were euploid. On the other hand, when the oocytes were cytoplasmic immature, $37 \%$ of the oocyte were normal (metaphase plate) and 50\% were chromosomally normal (table 1). When all the oocytes (metaphase II and unfertilized) were added, the global rate of aneuploidies in immature oocytes were significantly higher than mature $(P<0.05)$. The same issue occurs with the metaphase plate II disarrangements (table 2 ).

Two groups were conformed regarding the patients that performed ICSI and had sub-optimal fertilization $(<50 \%)$. The first group (A), cytoplasmic immaturity and sperm premature chromosome condensation (PCC) was the main feature observed in the failed-fertilized oocytes. The second group (B), sperm nuclear decondensation failure with normal cytoplasmic maturation status. Table 3 shows the population characteristics between both groups. No signif- icant differences were found regarding male and female age, sperm morphology, hormonal levels and AFC at follicular phase, dose of rFSH administered and days of ovarian stimulation. The number of oocytes retrieved after hCG was similar A vs. B (13.8 vs 12.9), similarly, the number of MII oocytes were similar (10.5 vs. 9.9) (table 4).

The percentage of top quality embryos (at $48 \mathrm{~h}$ ) formed from fertilized oocytes was significantly lower in group $A$ $(P<0.05)$, in addition the pregnancy rate was higher in group B (table 5).

\section{DISCUSSION}

Mammalian oocyte maturation comprises a series of complex mechanisms hormonally regulated. The competence of the human oocyte cannot be precisely predicted only with the observations made by the follicle size or nuclear maturation at the time of insemination or injection (Van Blerkom et al., 1997). The current work has not the objective to propose a competence oocyte marker (or markers), otherwise, intends give information about the correlation between a correct nuclear and cytoplasmic oocyte maturation and its adequate metaphase plate conformations and the aneuploidy rate (polar body). Also, is important to distinguish the impact of the cytoplasmic immaturity after ICSI and the reproductive outcomes in ART when this issue is compromise in fertilization failure or sub-optimal fertilization $(<50 \%)$.

As it is known, during ovarian stimulation for in vitro fertilization (IVF), it has been found a heterogeneous response in the oocyte cohort development, where an oocyte apparently normal, at metaphase II, cannot been fertilized for a competent sperm (Bedford \& Kim, 1993). Regarding to IVF cycles it has been described a fertilization failure after sperm penetration or a pre-implantation embryo arrest during their development (Van Blerkom, 1993; Van Blerkom et al., 1994). After ICSI, the heterogeneity of the oocyte cohort, from the point of view of their cytoplasmic maturity, has allowed to stablish a relation between the maturation status and fertilization failure (Alvarez Sedó et al., 2013).

The study of fertilization failure after ICSI allows understanding the different alterations of the normal fertilization mechanisms regarding to sperm and oocyte. Even though, it is true that ICSI technique was introduced as an alternative for severe male factor (allowing the sperm entry into the oocyte cytoplasm in a mechanical way avoiding a series of previous physiological events), this procedure has allowed the activation of the oocyte arrested at MII and continuing to their embryo development (Palermo et al., 1992). The oocyte activation begins when spermatozoa triggers a series of intracellular calcium pulses restarting and completing its meiosis. It can be determined some characteristics in the study of fertilization failure related with the sperm. The decondensation failure of the paternal genetic material (sperm DNA) is the most common. This disorder may be due to inability of the spermatozoa to activate the oocyte, either because of mutations and/or absence of the activation proteins or because of oocyte incapacity to remodel de sperm DNA (nuclear proteins removal). In relation with the oocyte responsibility in fertilization failure, is known that it can be implicated the cytoplasmic oocyte maturity, particularly the MPF (Schmiady et al., 1986). We have previously reported that the levels of pCdc2 (Y15) during oocyte maturation showed a variation of their expression according to the nuclear maturation. The pCdc2 (Y15) has a higher expression peak during anaphase-telophase I stages. This was consistent with other species about the levels MPF, which suffers a decrease during MI transition to MII (Alvarez Sedó, et al., 2013). The arrested oocyte at MII presents high levels of Cdc2 (T161) and the 
control mechanism does not allow the disruption of the arrest until the sperm entry. On the other hand, in the mature MII oocyte the levels of pCdc2 (Y15) are decrease. Therefore we stablished that the absence of this protein with a correct occurrence of the metaphase II and the first polar body, would indicate an optimal cytoplasmic and nuclear status.

This is the first report that describes the incidence of cytoplasmic immaturity and alterations in the metaphase plate conformation, moreover, with an increase of the aneuploidy rates in oocytes from ART cycles (controlled ovarian hyper stimulation). Regarding to this, it has been described the important role of the MPF and APC-C (anaphase promoting complex-Cyclosome). It means that a correct spindle check point function is associated with a correct MPF status and functionality (Gui \& Homer, 2012). Mad2, cohesin, kinetochores are part of the specific protein machinery that participates in this mechanism (Vogt et al., 2008). Numerous cytological and biochemical alterations occur in mammalian oocytes. Some of these changes can predispose cells to aneuploidy. The premature centromere separation (PCS) is now recognized as a predisposition to aneuploidy as well as nondisjunction. PCS involves separation of sister chromatids or homologues before anaphase onset; whereas, nondisjunction results from failure of sister chromatid or homologue separation at anaphase (Mailhes, 2008). Oocyte aging, ovarian stimulation and stress are some of the causes that are involved in the origin of these aberrations (Rodman, 1971; Angell et al., 1994; Mailhes et al., 1997; Steuerwald et al., 2005). These factors could be compromising the correct MPF activity triggering disarrangements at the APC checkpoint, resulting in oocyte aneuploidies. Alterations in human metaphase II spindle molecular motors could be also involved on these mechanisms, e.g. NuMA, Eg5. Our research group has previously reported that aged and stressed human oocytes could be associated to modifications in the expression and localization of NuMA and therefore implicated in oocyte aneuploidies (Alvarez Sedo et al., 2011). Regarding to patients with sub-optimal fertilization after ICSI, it's clearly seen that fertilized oocytes, from the cohort with cytoplasmic immaturity, had an impair quality evidenced by the poorest reproductive outcomes in comparison with those cycles where the main cause of fertilization failure was not oocyte cytoplasmic maturation. Particularly, in these cases, it could be possible that the entire oocyte cohort had quality impairment, but only few of them had the capacity of go beyond fertilization. However, these deficiencies could be evidenced during early cleavage.

\section{CONCLUSIONS}

Oocyte cytoplasmic immaturity is related to metaphase plate anomalies and aneuploidies. Fertilized oocytes, from a cohort with sub-optimal fertilization, with cytoplasmic immaturity, had poorer reproductive outcomes.

\section{CONFLICT OF INTERESTS}

No conflict of interest have been declared.

\section{Corresponding author:}

\section{Cristian Alvarez Sedó}

Centro de Estudios en Genética y Reproducción (CEGYR)

Viamonte 1438, C1055ABB, Buenos Aires, Argentina

E-mail: calvarez@cegyr.com

\section{REFERENCES}

Alvarez Sedó C, Schatten H, Combelles CM, Rawe VY. The nuclear mitotic apparatus (NuMA) protein: localization and dynamics in human oocytes, fertilization and early embryos. Mol Hum Reprod. 2011; 17:392-8. Alvarez Sedó C, Noblia F, Franco MC, Nodar F, Fiszbajn G, Papier S. Oocyte and sperm factors implicated in fertilization failure after ICSI. Fertil Steril. 2013; 100:S94.

Angell RR, Xian J, Keith J, Ledger W, Baird DT. First meiotic division abnormalities in human oocytes: Mechanism of trisomy formation. Cytogenet Cell Genet. 1994; 65:194-202.

Bedford M, Kim H. Sperm/egg binding patterns and oocyte cytology in a retrospective analysis of fertilization failures in vitro. Hum Reprod 1993;8:453-63.

Combelles C, Fissore R, Albertini D, Racowsky C. In vitro maturation of human oocytes and cumulus cells using a coculture three-dimensional collagen gel system. Hum Reprod $2005 ; 20: 1349-58$.

Downs SM. Protein synthesis inhibitors prevent both spontaneous and hormone-dependent maturation of isolated mouse oocytes. Mol Reprod Dev 1990;27:235-43.

Downs SM. Purine control of mouse oocyte maturation: evidence that nonmetabolized hypoxanthine maintains meiotic arrest. Mol Reprod Dev 1993;35:82-94.

Ferrell JE Jr. Xenopus oocyte maturation: new lessons from a good egg. Bioessays 1999;21:833-42.

Gui L, Homer H. Spindle assembly checkpoint signalling is uncoupled from chromosomal position in mouse oocytes. Development. 2012;139:1941-6.

Masui Y, Markert CL. Citoplasmic control of nuclear behavior during meiotic maturation of frog oocytes. J Exp Zool $1971 ; 177: 129-45$.

Mailhes JB, Young D, London SN. 1,2-Propanediol-induced premature centromere separation in mouse oocytes and aneuploidy in one-cell zygotes. Biol Reprod. 1997; 57:92-8.

Mailhes JB. Faulty spindle checkpoint and cohesion protein activities predispose oocytes to premature chromosome separation and aneuploidy. Environ Mol Mutagen. 2008; 49:642-58.

Moor RM, Mattioli M, Ding J, Nagai T. Maturation of pig oocytes in vivo and in vitro. J Reprod Fertil Suppl 1990;40:197-210.

Palermo G, Joris H, Devroey P. Pregnancies after intracytoplasmic injection of single spermatozoon into an oocyte. Lancet $1992 ; 4 ; 340: 17-18$.

Rodman TC. Chromatid disjunction in unfertilized ageing oocytes. Nature. 1971; 233:191-3.

Sagata N. [The roles of the Mos-MAPK pathway in oocyte meiosis and cellular transformation]. Tanpakushitsu Kakusan Koso 1996;41:1847-55.

Schmiady $H$, Sperling $K$, Kentenich $H$, Stauber M. Prematurely condensed human sperm chromosomes after in vitro fertilization (IVF). Hum. Genet 1986;74: 441-3.

Steuerwald NM, Steuerwald MD, Mailhes JB. Post-ovulatory aging of mouse oocytes leads to decreased MAD2 transcripts and increased frequencies of premature centromere separation and anaphase. Mol Hum Reprod. 2005;11:623-30. 
Thibault C. Hammond Memorial Lecture. Are follicular maturation and oocyte maturation independent processes? J Reprod Fertil 1977;51:1-15.

Van Blerkom, J. Development of human embryos to the hatched blastocyst stage in the presence or absence of a monolayer of Vero cells. Hum Reprod 1993;8:1525-39.

Van Blerkom J, Davis P, Merriam J. A retrospective analysis of unfertilized and presumed parthenogenetically activated human oocytes demonstrates a high frequency of sperm penetration. Hum Reprod 1994; 9:2381-8.

Van Blerkom J, Antczak M, Chrader R. The developmental potential of the human oocyte is related to the dissolved oxygen content of follicular fluid: association with vascular endothelial growth factor levels and perifollicular blood flow characteristics. Hum Reprod 1997; 12: 1047-55.

Verlhac MH, Kubiak JZ, Clarke HJ, Maro B. Microtubule and chromatin behavior follow MAP kinase activity but not MPF activity during meiosis in mouse oocytes. Development 1994;120:1017-25.

Vogt E, Kirsch-Volders M, Parry J, Eichenlaub-Ritter U. Spindle formation, chromosome segregation and the spindle checkpoint in mammalian oocytes and susceptibility to meiotic error. Mutat Res. 2008;651:14-29.

Whitaker M. Control of meiotic arrest. Rev Reprod 1996; :127-35. 